\title{
Some Advances in Hyperfibrinogenemia
}

\section{Shan Gao}

General Practitioner Department, the Affiliated Hospital of Southwest Medical University, Luzhou, Sichuan, 646000, China

\begin{abstract}
The normal content of human fibrinogen is $2 \sim 4 \mathrm{~g} / \mathrm{L}$, and if it is higher than $4 \mathrm{~g} / \mathrm{L}$, it is called hyperfibrinogenemia. The level of human plasma fibrinogen is related to coronary heart disease, acute cerebral infarction and femur head necrosis. For every $1 \mathrm{~g} / \mathrm{L}$ increase in fibrinogen levels, the risk of all these events will double. Therefore, fibrinogen levels should be related to the prevention and treatment of these diseases. After reviewing the recent literature, this paper summarizes the research progress of hyperfibrinemia.
\end{abstract}

\section{Keywords}

hyperfibrinogenemia; coronary disease; cerebral infarction; femur head necrosis

\section{高纤维蛋白原血症的某些进展}

高珊

\section{西南医科大学附属医院全科，中国・四川泸州 646000}

\section{摘 要}

人体纤维蛋白原的正常含量为 $2 \sim \mathrm{g} / \mathrm{L}$ ，如果高于 $4 \mathrm{~g} / \mathrm{L}$ ，则称为高纤维蛋白原血症。人体血浆纤维蛋白原水平与冠心病，急性 脑梗塞和股骨头坏死有关。纤维蛋白原水平每增加 $1 \mathrm{~g} / \mathrm{L}$, 所有这些事件的风险就会翻倍。因此, 纤维蛋白原水平应该与预防 和治疗这些疾病有关。在回顾了近期的文献后，本文总结了高纤维蛋白血症的研究进展。

关键词

高纤维蛋白原血症；冠心病；脑梗死；股骨头坏死

\section{1 高纤维蛋白血症与冠心病 (CHD )}

冠心病危害许多人的健康，并且仍然是发达国家的主要 死因。冠心病的传统危险因素包括高血压，高脂血症和吸烟。 通过有效控制这些因素，死亡率可降低 $50 \%$ 。随着对冠心 病认识的不断深人，凝血因子已成为许多研究的焦点。纤维 蛋白原和凝血因子及血管性血友病因子的增加可预测心血管 事件 ${ }^{[2]}$ 。异常的纤溶一凝血系统是血栓形成的重要因素。FIB 参与血液凝固过程, 是血栓形成的主要成分 ${ }^{[3]}$ 。

纤维蛋白原与冠心病的发生和发展密切相关。血浆纤维 蛋白原浓度的显着增加可以促进冠状动脉弹样硬化的发展和 进展。纤维蛋白原参与血管内血栓的形成，其在血栓形成过 程中起重要作用。血浆纤维蛋白原水平升高会增加血液粘度, 诱导血小板活性，增加血小板聚集，减少血液流动和血液。 流变学的变化进一步降低了血管壁切断的速度，从而促进了 动脉码样硬化斑块和血栓的形成。纤维蛋白原及其降解产物
在冠心病的发生和发展中起重要作用, 并且还与纤维斑块的 稳定性和急性冠状动脉事件的发生有关。研究表明, 高纤维 蛋白原可能与冠状动脉病变的数量和冠状动脉病变的严重程 度有关, 纤维蛋白原可用作冠状动脉心血管事件的独立预测 因子。在对 100 名冠心病患者进行的为期 3 个月的随访调查中, 高纤维蛋白原患者的心血管事件发生率显着高于低纤维蛋白 原患者。研究结果显示, 冠心病患者的血浆纤维蛋白原水平 明显高于正常人的平均水平, 并且通过比较冠心病患者的纤 维蛋白原水平与不同程度的冠状动脉疾病, 结果发现, 患者 血浆中纤维蛋白原含量较高, 冠状动脉病变血浆纤维蛋白原 含量与冠状动脉病变严重程度的 Pearson 相关性分析显示血浆 纤维蛋白原含量与冠状动脉病变呈显着正相关。（ $\mathrm{r}=0.559$, $\mathrm{P}<0.05$ ) 急性心肌梗死和不稳定型心绞痛患者纤维蛋白原水 平明显高于稳定型心绞痛患者。这种纤维蛋白原水平可能有 助于识别患有冠心病的高风险患者。纤维蛋白原对冠心病患 者纤维斑块的稳定性有一定的影响, 这与冠状动脉的炎症反 
应有关。它对预测心血管相关病变的程度具有指导意义。

\section{2 高纤维蛋白血症与急性脑梗死}

纤维蛋白原 $(\mathrm{Fg})$ 斑块与各种炎性神经变性疾病如阿尔 茨海默病, 多发性硬化, 中风和创伤性脑损伤中的记忆丧失 有关。然而，纤维蛋白原在神经血管单元中的作用机制尚不 清楚。纤维蛋白原水平升高可能对急性缺血性卒中患者的生 存产生不利影响, 血浆纤维蛋白原也可用作急性缺血性卒中 的预测因子。急性缺血性中风，在临床上也称为脑梗塞，是 临床神经系统的疾病, 在中老年人群中是常见的。残疾率和 死亡率都很高。血浆纤维蛋白原具有凝血作用。中国研究表明, 人体血浆纤维蛋白原含量在血栓前会明显增加, 这会直接增 加血浆粘度, 最终导致血栓形成 ${ }^{[4]}$ 。由于急性缺血性中风给 患者, 家属和社会带来了很多负担, 因此它是检测和预防疾 病的最有效方法。纤维蛋白原是所有部位血管内血栓形成的 主要蛋白质成分。纤维蛋白原的形成和功能是生理性止血, 并且也是病理性血栓形成所必需的。纤维蛋白原水平预测动 脉和静脉血栓栓塞事件的复发。一旦血浆中的纤维蛋白水平 增加，它可能增加血液的粘度，促进血小板聚集，并对心血 管和脑血管系统产生不利影响。研究表明纤维蛋白原可能参 与心血管和脑血管疾病的形成。此外, 研究结果还表明, 在 急性脑梗死的诊断中，单独使用纤维蛋白原对急性脑梗死的 诊断最为敏感 ( $92.0 \%), C$ 反应蛋白的敏感性最高 $(85.7 \%)$ 。 蛋白质, C- 反应蛋白, 纤维蛋白原和尿酸的诊断特异性可高 达 $96.0 \%$, 可获得较高的应用价值。因此, 认为纤维蛋白原 检测有助于了解急性脑梗死的进展情况，联合检测具有较大 的应用价值。

\section{1 纤维蛋白原对急性缺血性脑卒中患者生存的影响}

不同纤维蛋白原水平对急性缺血性脑卒中严重程度的影 响在对 78 例急性缺血性脑卒中患者的研究中, 比较了不同纤 维蛋白原水平组间急性缺血性脑卒中严重程度。

$[n(\%)]$

\begin{tabular}{lcccc}
\hline 组别 & $n$ & 重度 & 中度 & 轻度 \\
\hline 高纤维蛋白原血症组 & 39 & $24(61.54)$ & $13(33.33)$ & $2(5.13)$ \\
血纤维蛋白原正常组 & 39 & $11(28.21)$ & $5(12.82)$ & $23(58.97)$ \\
$\chi^{2}$ & & 5.381 & 4.876 & 5.024 \\
$P$ & & 0.021 & 0.032 & 0.025 \\
\hline
\end{tabular}

急性缺血性卒中的严重程度在高纤维蛋白原血症组和正
常纤维蛋白原血症组中是不同的, 在高纤维蛋白原血症组中 重度和中度的患者具有统计学意义。

\section{2 血浆纤维蛋白原水平的不同对患者出院治疗结局} 的影响

\begin{tabular}{lccc}
\hline 组别 & $n$ & 结局良好 & 结局不良 \\
\hline 高纤维蛋白原血症组 & 39 & $11(28.21)$ & $28(71.79)$ \\
血纤维蛋白原正常组 & 39 & $31(79.49)$ & $8(20.51)$ \\
$\chi^{2}$ & & & \multicolumn{2}{c}{4.876} \\
$P$ & & & \multicolumn{2}{c}{0.032} \\
\hline
\end{tabular}

高纤维蛋白原血症组患者的治疗结局不良发生率高于血 纤维蛋白原正常组患者。

\section{3 血浆纤维蛋白原水平对患者治愈率以及病死率}

\section{比较}

\begin{tabular}{lccc}
\hline 组别 & $n$ & 治息率 & 病死率 \\
\hline 高纤维蛋白原血症组 & 39 & $30(76.92)$ & $9(23.08)$ \\
血纤维蛋白原正常组 & 39 & $37(94.87)$ & $2(5.13)$ \\
$\chi^{2}$ & & 3.217 & 4.876 \\
$P$ & & 0.060 & 0.032 \\
\hline
\end{tabular}

血纤维蛋白原正常组患者出院后的恢复效果强于高纤维 蛋白原血症组, 虽然两组治愈率差异无统计学意义 $(\mathrm{P}>0.05)$, 但前者的死亡率低于后者。差异有统计学意义 $(\mathrm{P}<0.05)$ 。 ${ }^{[5]}$ 血浆纤维蛋白是一种重要的凝血因子, 其在人体内的含量在 血栓前会显着增加。 ${ }^{[6]}$ 纤维蛋白原水平升高可能对急性缺血 性卒中患者的生存产生不利影响, 血浆纤维蛋白原也可能是 急性缺血性卒中的预测因子。

\section{3 高纤维蛋白原血症与非创伤性股骨头坏死}

非创伤性股骨头坏死的发生与使用大量酒精和使用大剂 量的内固醇激素密切相关。使用大剂量激素引起的股骨头坏 死的发生率在非创伤性股骨头坏死中占第一位。临床中长期 服用内固醇激素的患者大多含有糖尿病、系统性红斑狼疮、 类风湿关节炎、器官移植等基础病, 这些疾病可造成血管内 皮损伤, 从而导致机体处于高凝状态, 使得血浆中纤维蛋白 原含量增高。在动物实验中表明血塞通、脂必妥和阿仑膦酸 钠与类同醇激素同时使用可增加实验动物股骨头血流量, 改 善血液流变学状况, 预防或减缓股骨头坏死的发生。

\section{4 小结}

高纤维蛋白血症主要是纤维蛋白原升高, 在检验里属于 
综述性文章
Review

血浆凝血酶原时间，目前大多数医院的急诊脑卒中中心急查 项目为血常规、电解质、肾功、凝血、 PT、头胸 CT 来判断 脑卒中患者是否有溶栓指征。但是对于冠心病及股骨头坏死 病人则很少检测纤维蛋白原含量, 以此来预防和治疗疾病。

\section{参考文献}

[1] FORD E S,AJANI U A,CROFT J B,et al.Explaining the decrease in U.S.deaths from coronary disease, $1980-2000[\mathrm{~J}] . \mathrm{N}$ Engl Med,2007,356(23):2388-2398

[2] LACEY B,HERRINGTON W G,et al.The role of emerging risk factors in cardiovascular outcomes[J].Curr Atherosvler
亚洲临床医学杂志・第 02 卷・第 03 期・ 2019 年 08 月 DOI: https://doi.org/10.26549/yzlcyxzz.v2i3.2413

Rep, 2017,19(6):28-38.

[3] LOWE G D.Fibrinogen assays for cardiovascular risk assessent[J]. Clin Chem,2010,56(5):693-695.

[4] 凌倚峰, 程忻, 杨璐萌, 等.急性缺血性卒中患者静脉溶栓治疗的 临床预后影响因素分析 [J]. 中华神经科杂志 ,2014,47(7):449-454

[5] 严兴亚, 陈然, 吕鸿燕等.Dragon 评分对急性缺血性卒中静脉溶栓 近期疗效的预测价值 [J]. 中风与神经疾病杂志 , 2016,33(12):11181121.

[6] 张瑜, 赵德臣, 刘婵娟, 任春娜. 血纤维蛋白原对急性缺血性卒中 患者生存状况的影响 [J]. 中国医药科学 ,2018,8(15):135-137. 